\title{
Features of the Quasi-Static and Dynamic Magnetization Switching in NiO Nanoparticles: Manifestation of the Interaction between Magnetic Subsystems in Antiferromagnetic Nanoparticles
}
D.A. Balaev ${ }^{1,2}$,
A.A. Krasikov ${ }^{1}$,
S.I. Popkov ${ }^{1,2}$,
A.A. Dubrovskiy ${ }^{1,2}$,
S.V. Semenov ${ }^{1,2}$
D.A. Velikanov ${ }^{1}$, V.L. Kirillov ${ }^{3}$, and O.N. Martyanov ${ }^{3}$

${ }^{1}$ Kirensky Institute of Physics, Federal Research Center "Krasnoyarsk Scientific Center, Siberian Branch, Russian Academy of Sciences”, Krasnoyarsk, 660036 Russia

${ }^{2}$ Siberian Federal University, Krasnoyarsk 660041, Russia

${ }^{3}$ Boreskov Institute of Catalysis, Siberian Branch, Russian Academy of Sciences, Novosibirsk, 630090 Russia

\begin{abstract}
We report on the investigations of a system of 8-nm $\mathrm{NiO}$ particles representing antiferromagnetic (AFM) materials, which are weak magnetic in the form of submicron particles, but can be considered to be magnetoactive in the form of nanoparticles due to the formation of the uncompensated magnetic moment in them. The regularities of the behavior of magnetization switching in AFM nanoparticles are established by studying the magnetic hysteresis loops under standard quasi-static conditions and in a quasi-sinusoidal pulsed field of up to $130 \mathrm{kOe}$ with pulse lengths of 4-16 ms. The magnetic hysteresis loops are characterized by the strong fields of the irreversible magnetization behavior, which is especially pronounced upon pulsed field-induced magnetization switching. Under the pulsed field-induced magnetization switching conditions, which are analogous to the dynamic magnetic hysteresis, the coercivity increases with an increase in the maximum applied field $H_{0}$ and a decrease in the pulse length. This behavior is explained by considering the flipping of magnetic moments of particles in an external ac magnetic field; however, in contrast to the case of single-domain ferro- and ferrimagnetic particles, the external field variation rate $d H / d t$ is not a universal parameter uniquely determining the coercivity. At the dynamic magnetization switching in AFM nanoparticles, the $H_{0}$ value plays a much more important role. The results obtained are indicative of the complex dynamics of the interaction between different magnetic subsystems formed in AFM nanoparticles.
\end{abstract}

\section{Introduction}

The diversity of magnetic nanoparticle-based materials includes a vast family of antiferromagnetic (AFM) nanoparticles. A bulk antiferromagnet is a weak magnetic substance, whereas AFM nanoparticles strongly influenced by defects [1-3] and surface effects are characterized by the formation of additional magnetic subsystems [4-12]. This is reflected in a drastic difference between the magnetic properties of bulk antiferromagnets and AFM nanoparticles. The main feature of AFM nanoparticles is the occurrence of an uncompensated magnetic moment; in these materials, in fact, a ferromagnetic (FM) subsystem forms [6-23], which becomes especially important in particles 10-20 $\mathrm{nm}$ in size and smaller. This significantly broadens the range of possible applications of AFM nanoparticles, which, in contrast to the bulk analog or submicron AFM particles, are already «magnetic».

In addition, in AFM nanoparticles, similar to ferri- and ferromagnetic ones, the developed surface and broken bonds give rise to the well-known effects accompanying a spin-glass state of surface spins [4-6, 24-32]. Thus, already three magnetic subsystems can coexist in an AFM nanoparticle, which are the antiferromagnetically ordered core, the FM subsystem (the uncompensated magnetic moment of a particle), and the subsystem of surface spins. The interaction 
between these subsystems causes many interesting effects observed in the magnetic properties of the AFM nanoparticle-based materials. The elucidation of features of the magnetic behavior of AFM nanoparticles makes it possible to establish the mechanisms of the interaction between the magnetic subsystems in these magnetically complex objects.

Among a vast set of available experimental techniques for studying the magnetic state of nanoparticles, very promising is the examination of the dynamic magnetic hysteresis (DMH). In this method, the effect of frequency and amplitude of an external ac field on the parameters of the magnetic hysteresis loop is studied. In multidomain submicron particles (or other objects, for example, magnetic films), the parameters of the DMH loop are determined, to a great extent, by the motion of domain walls, while in single-domain FM particles, the dynamic hysteresis is related to the effect of the magnetization switching time on the flipping of magnetic moment of a particle [33-37]. The investigations of the $\mathrm{DMH}$ in systems of magnetic nanoparticles become very important due to the hyperthermia effect [38-43]. Here, it is necessary to understand the effect of the internal properties, including the magnetic anisotropy with its surface contribution, particle size, and features of the magnetic ordering inside a particle [44], on the parameters of the DMH loop, specifically, the coercivity $H_{\mathrm{C}}$, remanent magnetization, and hysteresis loop area.

There is a circumstance that is of crucial importance in studying the DMH. In conventional facilities for generation of the ac magnetic field $H=H_{0} \sin (2 \pi v t)$, serios limitation are imposed on the maximum field $H_{0}$ and frequency $v$. These limitations are related to the power released in a solenoid. Therefore, at frequencies of about $10^{2}-10^{3} \mathrm{~Hz}$, it is difficult to obtain the fields $H_{0}$ stronger than $\sim 10^{3}$ Oe. This significantly complicates the investigations of the DMH in the materials with the high magnetic anisotropy, since, if the $H_{0}$ value will be lower than the field $H_{\text {irr }}$ of the irreversible magnetization behavior, then the resulting hysteresis loop will be minor. Hence, the obtained parameters $\left(H_{\mathrm{C}}\right.$ and the remanent magnetization) will be mainly determined by the effects of the minor hysteresis loop, which makes it difficult to understand the effect of the internal properties on the DMH features.

The use of pulsed fields is an alternative to the conventional techniques for studying the DMH $[44,45]$. In this case, the power release in a solenoid is limited to a single external field variation cycle; therefore, the maximum applied field can be increased to hundreds of kilooersted. Using this approach, the loops of pulsed field-induced magnetization switching in a high-coercivity material containing $\varepsilon-\mathrm{Fe}_{2} \mathrm{O}_{3}$ nanoparticles were investigated [45] (the field $H_{\text {irr }}$ for $\varepsilon-\mathrm{Fe}_{2} \mathrm{O}_{3}$ attains $\sim 60 \mathrm{kOe}$ ).

In AFM nanoparticles, the presence of an FM subsystem causes, under certain conditions, the existence of the magnetization hysteresis $[4,7,8,12,20-22,27-30,46]$; consequently, the hysteresis will be observed also under the dynamic magnetization switching. Many theoretical studies have been devoted to the DMH in FM [35, 36, 47-50] and AFM nanoparticles [51-53], whereas the available experimental works only deal with FM nanoparticles. It is well-known that AFM nanoparticles are characterized by the strong (about $10^{5} \mathrm{Oe}$ ) fields of the irreversible behavior of the magnetization curve $[46,54,20]$. Here, the pulsed technique for studying the dynamic magnetization switching is advantageous as well, since it covers an essentially broader range of the $H_{0}$ amplitude than the conventional loop-scope methods. The results of the preliminary investigations of $\mathrm{NiO}$ [55] and ferrihydrite [56] nanoparticles showed a significant difference between the effects of parameters of the pulsed field ( $H_{0}$ and pulse length) on the coercivity in these antiferromagnetically ordered nanoparticles and FM ones. This behavior is apparently the manifestation of the interaction between the magnetic subsystems in AFM nanoparticles. This study is devoted to the detailed examination of the quasi-static magnetic properties and effect of the pulsed field-induced magnetization switching on the coercivity of $\mathrm{NiO}$ AFM nanoparticles in a wide temperature range (in above-cited studies [55, 56], the measurement range was restricted to $78 \mathrm{~K}$ ). It is demonstrated by the example of $\mathrm{NiO}$ nanoparticles that the interaction between the magnetic subsystems in an AFM nanoparticle leads to the nontrivial behavior of the magnetic properties of such objects. 


\section{Experimental}

\subsection{Synthesis and characterization of the nanosized $\mathrm{NiO}$ compound}

$\mathrm{NiO}$ nanoparticles were synthesized by thermal decomposition of nickel oxalate $\mathrm{NiC}_{2} \mathrm{O}_{4}$. $2 \mathrm{H}_{2} \mathrm{O}$. The decomposition procedure included the temperature rise to $400^{\circ} \mathrm{C}$ for $40 \mathrm{~min}$ and the 10 min exposure at this temperature.

A reference sample of submicron nickel oxide particles was the ultra-high purity $\mathrm{NiO}$ reagent hereinafter referred to as bulk $\mathrm{NiO}$.

The X-ray diffraction (XRD) pattern of the sample under study was obtained on a Bruker D8 Advance X-ray diffractometer (Germany) in $\mathrm{CuK \alpha}$ radiation at $\lambda=1.5418 \AA$ (Fig. 1). All the diffraction peaks correspond to the NiO phase (PDF No. 047-1049). The NiO unit cell parameter coincides with a standard value (sp. gr. $F m \overline{3} m, a=b=c=4.176 \AA$, and $\alpha=\beta=\gamma=90^{\circ}$ ). The coherent scattering region determined from the diffraction peak broadening is about $9 \mathrm{~nm}$.

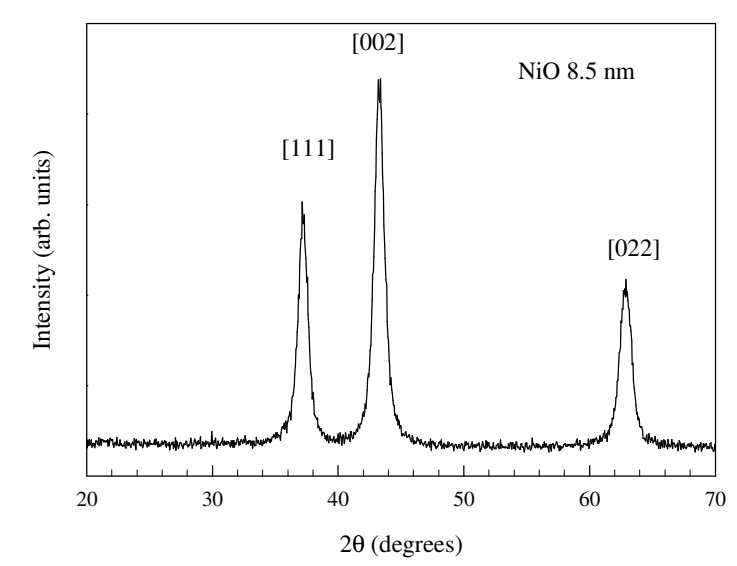

Fig. 1. XRD pattern of the investigated $\mathrm{NiO}$ nanoparticle sample.

The transmission electron microscopy (TEM) investigations were carried out on a Hitachi HT7700 transmission electron microscope at an accelerating voltage of $100 \mathrm{kV}$. The specimens were prepared using a conventional technique, specifically, by the deposition of $\mathrm{NiO}$ nanoparticles suspended in alcohol and preliminary dispersed in an ultrasonic bath onto carbon grids. Figure 2 shows a typical microphotograph of the investigated sample. Inset in Fig.2 presents a nanoparticle size distribution histogram. The average size of $\mathrm{NiO}$ nanoparticles was found to be $<\mathrm{d}\rangle \approx 8.5 \mathrm{~nm}$, which is consistent with the coherent scattering region determined from the X-ray diffraction data (Fig. 1). 


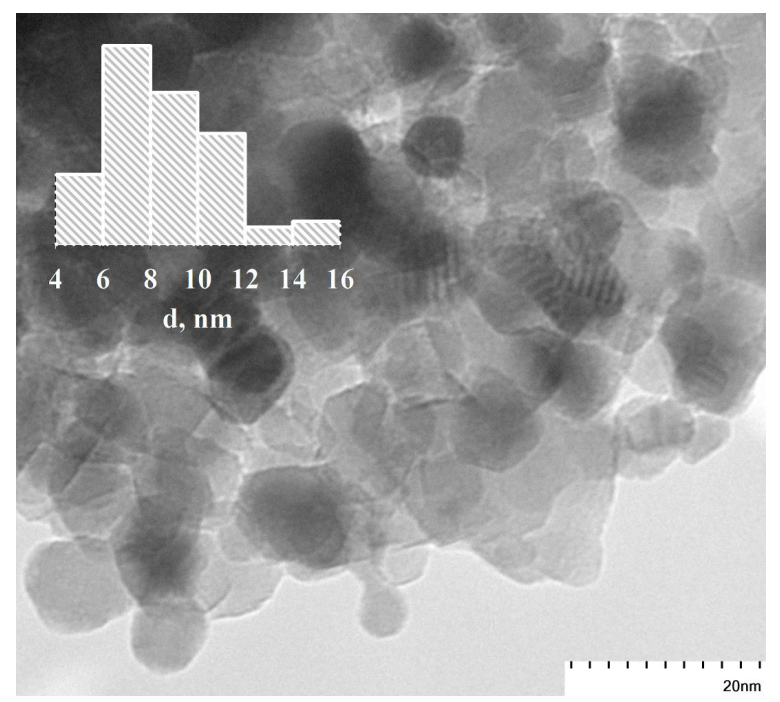

Fig. 2. TEM micrograph of nanoparticles of the investigated sample and particle size distribution histogram.

\subsection{Measurements of the quasi-static magnetic properties}

The temperature dependences of the magnetization $(M(T))$ were measured on a SQUID magnetometer [57] upon zero field cooling (the ZFC mode) and cooling in a field (the FC mode).

Quasi-static magnetic hysteresis loops were measured on original [58] and Quantum Design PPMS-6000 vibrating sample magnetometers (VSM). The sample was cooled in zero field. The field variation rate $(d H / d t)_{\text {VSM }}$ during the measurements of the quasi-static $M(H)$ hysteresis loops was $\sim 50 \mathrm{Oe} / \mathrm{s}$. To obtain the dependence of the $H_{\mathrm{C}}$ value on the maximum applied field $H_{0}$, a set of minor hysteresis loop was measured in the gradually increasing field $H_{0}$. After the measurements at a certain temperature, the sample was warmed up to room temperature to cancel the thermomagnetic prehistory.

In all the measurements of the magnetic properties, the $\mathrm{NiO}$ nanoparticle (powder) sample was fixed in paraffine.

\subsection{Measurements of the magnetic hysteresis loops in pulsed fields}

The measurements were performed on an original pulsed field generation setup developed at the Kirensky Institute of Physics, Siberian Branch, Russian Academy of Sciences [59]. The setup is based on the capacitor battery discharge through a solenoid; the controlled capacitor charging voltage determines the maximum field $H_{0}$. The setup is an LCR contour in which the capacitor battery banks can be switched to different capacitances, thereby varying the pulse length. The oscillatory process in such setups is usually stopped by a thyristor after the first half-wave, while the measurements of the magnetization hysteresis loops requires no less than two half-waves. To form the second half-wave, diodes were included counter-parallel to the thyristor in the setup circuit.

Typical time dependences of the field in the solenoid $(H(t))$ at different fields $H_{0}$ and pulse lengths (half-wave time $\tau_{\mathrm{P}}$ ) are presented in Fig. 3. The zero filed at the instant of time $t=\tau_{\mathrm{P}}$ is caused by closing the thyristor units. In this work, the measurements were performed at $\tau_{\mathrm{P}}$ values of 4, 8, and $16 \mathrm{~ms}$; the $H_{0}$ value was ranged from 30 to $130 \mathrm{kOe}$. For the operation modes used, the $H(t)$ are described with good accuracy by the harmonic law

$$
H(t)=H_{0} \sin \left(t / 2 \tau_{\mathrm{P}}\right)
$$

with regard to the damping. 
The magnetization was measured with an inductance transducer representing a system of coaxial compensated coils in which the sample was placed. The signal induced in the coils was amplified and detected by a digital storage oscilloscope. The measurements were performed in the temperature range of 80-300 K. At constant temperature, the $H_{0}$ value in each next measurement was higher than the previous value. After the measurement at a certain temperature, the sample was warmed up to room temperature. The results obtained by the pulsed technique were compared in the absolute value with the VSM data.

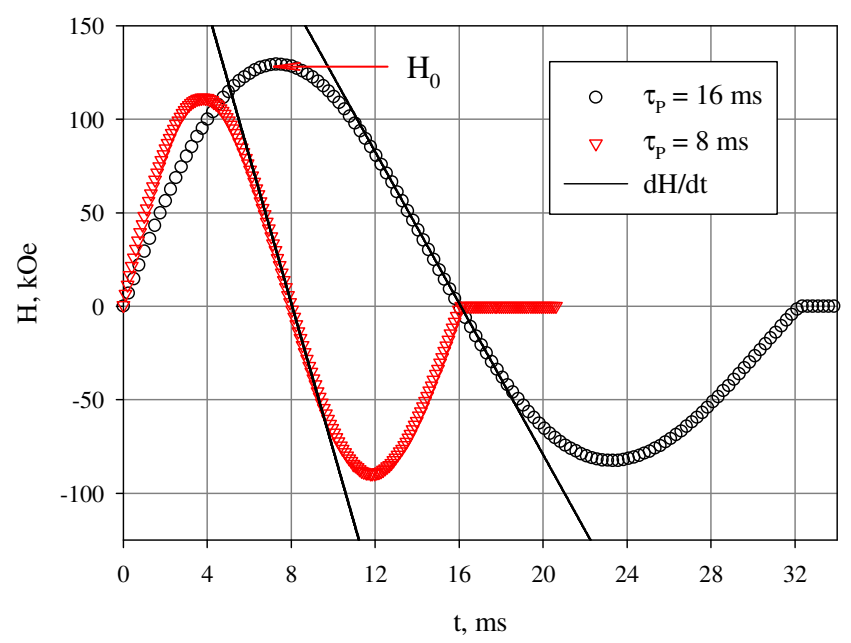

Fig. 3. Typical time dependences of the solenoid field in the pulsed technique at different maximum applied fields $H_{0}$ and indicated pulse lengths $\tau_{\mathrm{P}}$. The slopes of the straights correspond to the field variation rate $d H / d t$ at the instant of magnetization switching of the sample.

\section{Quasi-Static Magnetic Properties}

\subsection{Temperature dependences of magnetization $M(T)$}

In Fig. 4, the temperature dependences of the magnetization of the investigated sample, together with the data for bulk $\mathrm{NiO}$, are shown in the form of the $M(T) / H$ data. The ZFC dependences and the FC dependences obtained in fields of $H=100 \mathrm{Oe}$ and $1 \mathrm{kOe}$ are presented. The pronounced $M(T)_{\mathrm{ZFC}}$ maxima and the discrepancy between the $M(T)_{\mathrm{ZFC}}$ and $M(T)_{\mathrm{FC}}$ dependences with decreasing temperature are observed. As the external field increases, the temperature $T_{\max }$ shifts toward lower temperatures. The $M / H$ value decreases with increasing field and significantly exceeds the value for the bulk NiO sample. The data obtained point out the superparamagnetic (SPM) blocking with a decrease in temperature, which is typical of magnetic nanoparticles. In our case, the uncompensated magnetic moments of particles are blocked. The presence of particle moments (the FM subsystem) ensures the higher $M / H$ value than in the bulk antiferromagnet (bulk NiO). The $T_{\max }$ value at $H=100 \mathrm{Oe}$ is $\sim 185 \mathrm{~K}$, which is consistent with the data obtained in $[30,60,61]$ for $\mathrm{NiO}$ nanoparticles of similar size. 


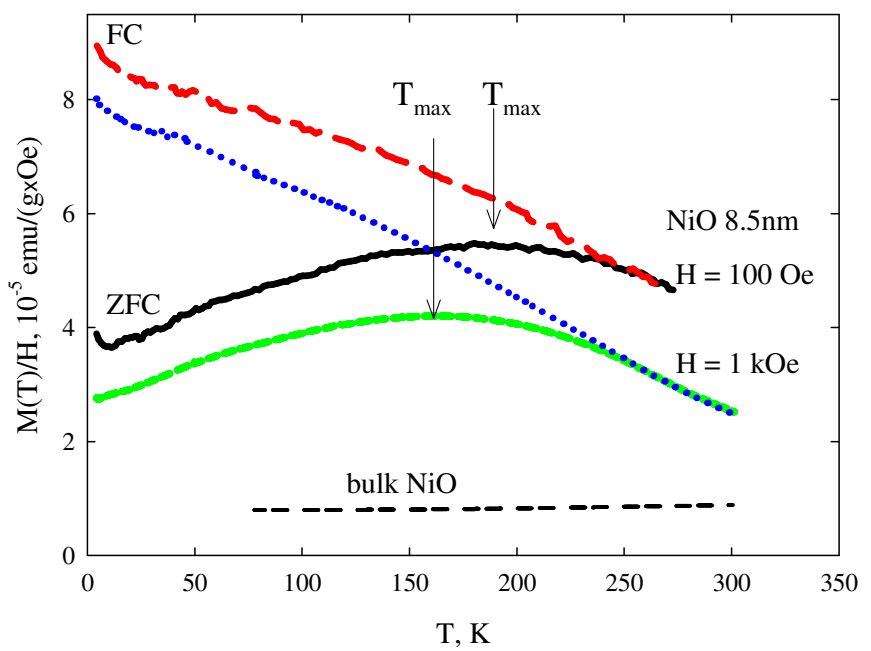

Fig. 4. Temperature dependences of $M(T) / H$ under the ZFC conditions and in the FC mode in fields of $H=0.1$ and $1 \mathrm{kOe}$ for the investigated sample of $\mathrm{NiO}$ nanoparticles with an average size of $8.5 \mathrm{~nm}$ and for submicron $\mathrm{NiO}$ nanoparticles (bulk $\mathrm{NiO}$ ).

\subsection{Magnetic hysteresis loop of the investigated samples}

Let us consider the behavior of the magnetization curves in the temperature region where the magnetic moments of particles (the FM subsystem) are blocked. Figure 5 shows the $M(H)$ dependences of the investigated sample of $\mathrm{NiO}$ particles at temperatures of 4.2 and $80 \mathrm{~K}$. The maximum external field $H_{0}$ was $60 \mathrm{kOe}$. The shape of the magnetization curve is approximately described by the field-linear contribution, which is pronounced in strong fields, and by the fieldirreversible function with the remanent magnetization and coercivity. As was mentioned in [62, 63, 9, 10], in AFM nanoparticles, the field-linear contribution has a slope, which exceeds by far the susceptibility of the corresponding bulk material. This can be seen in Fig. 5, which shows also the data for the bulk NiO sample. The slope of the $M(H)$ dependences of the bulk NiO compound, which is only determined by the AFM susceptibility of $\mathrm{NiO}$, is much smaller than the slope of the linear contribution of the $M(H)$ dependence of nanoparticles. This allows us to conclude that, as was mentioned in Introduction, the nanoparticles include several magnetic subsystems. Obviously, the FM subsystem in the blocked state ensures the main contribution to the hysteretic behavior of the magnetization curve. Along with the antiferromagnetically ordered particle core, there exists one more subsystem. It would be reasonable to believe that this contribution is determined by the subsystem of surface spins, which, in the low-temperature region, can be in the spin glass state [26-32] and, at high temperatures, exhibits the paramagnetic behavior [32, 10, 64-66]. Note that the spin glass behavior can also be characterized by the irreversibility of the magnetization curve [24, 25] and therefore each subsystem can contribute to the $M(H)$ hysteresis in nanoparticles below the temperature of the transition to the spin glass state. 


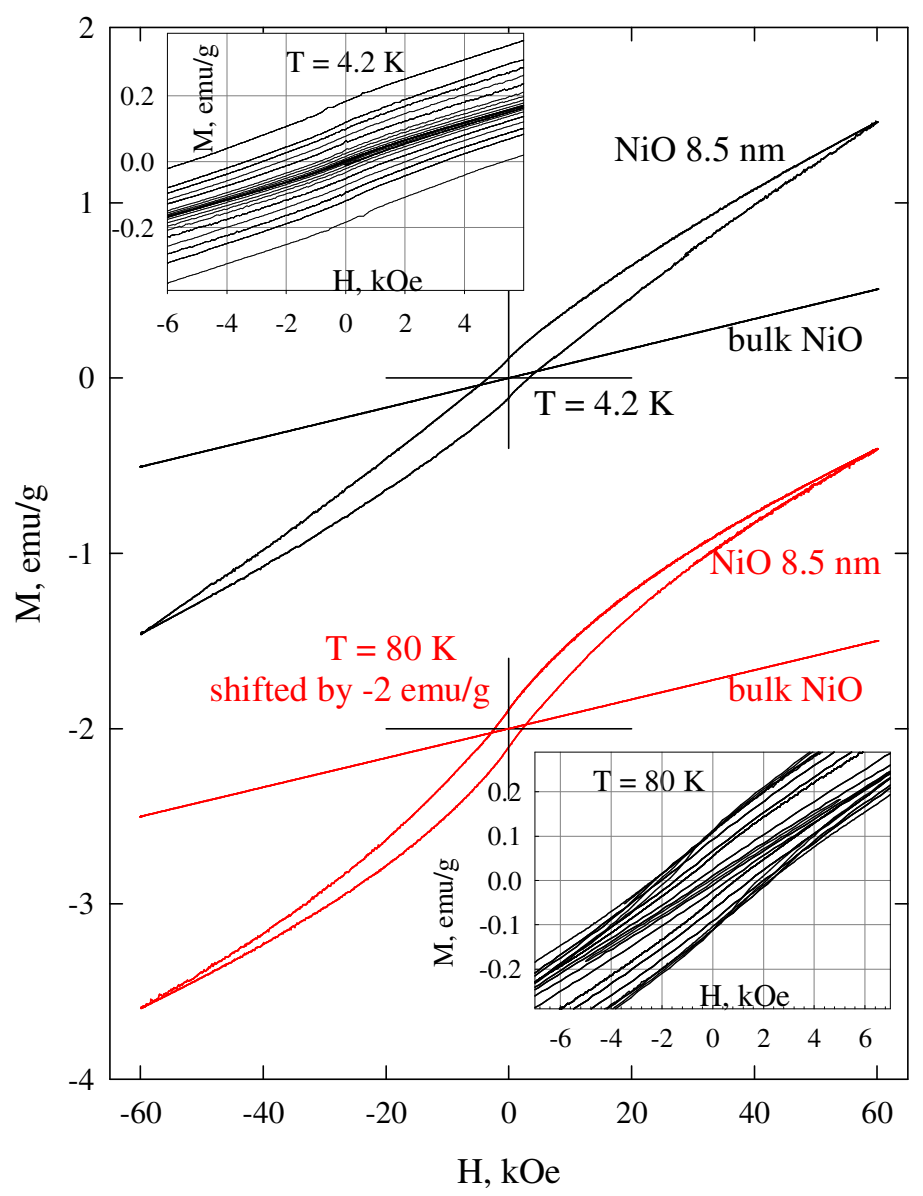

Fig. 5. VSM magnetic hysteresis loops for the investigated $\mathrm{NiO}$ nanoparticle sample and for submicron $\mathrm{NiO}$ particles (bulk $\mathrm{NiO}$ ) at temperatures of 4.2 and $80 \mathrm{~K}$ (then data obtained at $T=80 \mathrm{~K}$ are shifted along the ordinate axis by $-2 \mathrm{emu} / \mathrm{g}$ ). Insets: families of the minor hysteresis loops of $\mathrm{NiO}$ nanoparticles up to a maximum field of $90 \mathrm{kOe}$ near the origin of coordinates.

It can be seen in Fig. 5 that the magnetic hysteresis loops remain open at the indicated data in fields $H_{0}$ of up to $60 \mathrm{kOe}$. This is additionally confirmed by the data in the upper inset in Fig. 5, which shows the portions of the minor $M(H)$ hysteresis loops at $T=4.2 \mathrm{~K}$ near the origin of coordinates obtained at a gradual increase in the $H_{0}$ value to $75 \mathrm{kOe}$. Both the width and the height of the loops grow with $H_{0}$. The portions of the minor $M(H)$ hysteresis loops in fields $H_{0}$ of up to $80 \mathrm{kOe}$ for a temperature of $80 \mathrm{~K}$ are shown in the lower inset in Fig. 5. Here, we may state that, at sufficiently large $(70-80 \mathrm{kOe}) H_{0}$ values, the portions of the minor loops are already close to each other and, at these maximum fields, the $M(H)$ dependence is similar to the limiting hysteresis loop. As was shown in [46, 54, 20], antiferromagnetically ordered ferrihydrite nanoparticles exhibit the high irreversibility fields and, therefore, very strong external fields are required to obtain the limiting hysteresis loop. A similar picture is observed for $\mathrm{NiO}$ nanoparticles, which points out the universality of such a behavior of AFM nanoparticles.

Using the family of minor loops, we can obtain the dependences of the coercivity on the maximum applied field $\left(H_{\mathrm{C}}\left(H_{0}\right)\right)$. Figure 6 presents these data for different temperatures. At the sufficiently high temperatures $(80$ and $120 \mathrm{~K})$, the $H_{\mathrm{C}}\left(H_{0}\right)$ dependences are $S$-shaped and have a plateau, which, in agreement with the data from Fig. 5 (see above), shows that, at $H_{0} \sim 80 \mathrm{kOe}$, the $M(H)$ loops, are, in fact, limiting. At the same time, the data in Fig. 5 demonstrate that, at low 
temperatures, the field $H_{0} \sim 80 \mathrm{kOe}$ is obviously insufficient even to see a trend to the plateau in the $H_{\mathrm{C}}\left(H_{0}\right)$ dependence.

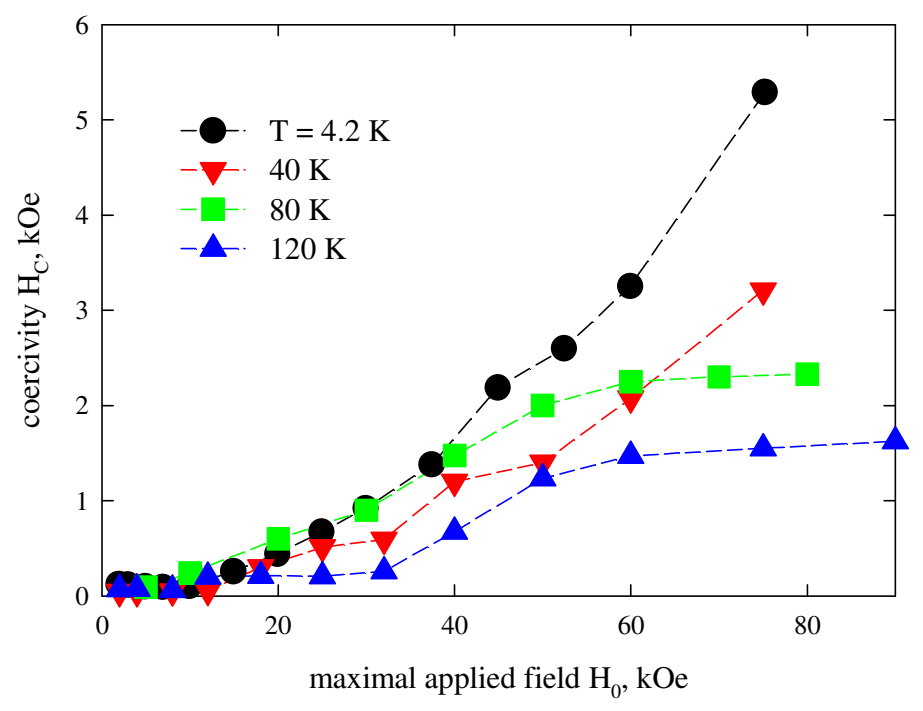

Fig. 6. Dependences of coercivity $H_{\mathrm{C}}$ on maximum applied field $H_{0}$ for the NiO nanoparticle sample obtained from the family of minor hysteresis loops at different temperatures.

In [54], the shape of the $H_{\mathrm{C}}\left(H_{0}\right)$ dependence of ferrihydrite nanoparticles and the strong fields at which this dependence achieves a plateau were explained by the effect of high barriers induced by the magnetic anisotropy. Reasonably, a source of the additional magnetic anisotropy can be the interaction between the above-mentioned magnetic subsystems in AFM nanoparticles. With an increase in temperature, this interplay between the magnetic subsystems will obviously manifest itself weaker, the field of the irreversible behavior of the magnetization will decrease, and the $H_{\mathrm{C}}\left(H_{0}\right)$ dependence will achieve a plateau at lower $H_{0}$ values.

\section{Magnetization Switching in a Pulsed Field}

\subsection{Magnetic hysteresis loops in a pulsed field}

Figure 7 shows typical hysteretic $M(H)$ dependences for the investigated samples (at $T=80$, 150 , and $200 \mathrm{~K}$ ). Here, the quasi-static magnetometry data (the maximum applied field $H_{0}=60 \mathrm{kOe}$ ) are presented together with the $M(H)$ dependences obtained in pulsed fields at $\tau=8 \mathrm{~ms}$ and $\mathrm{H}_{0} \approx 70 \mathrm{kOe}$ and $115 \mathrm{kOe}$. It can be seen that the irreversibility of the magnetization curves becomes more pronounced during the pulsed measurements (see the $M(H)$ portions in strong fields. At the same time, as the temperature increases, the $M(H)$ irreversibility becomes less pronounced. 


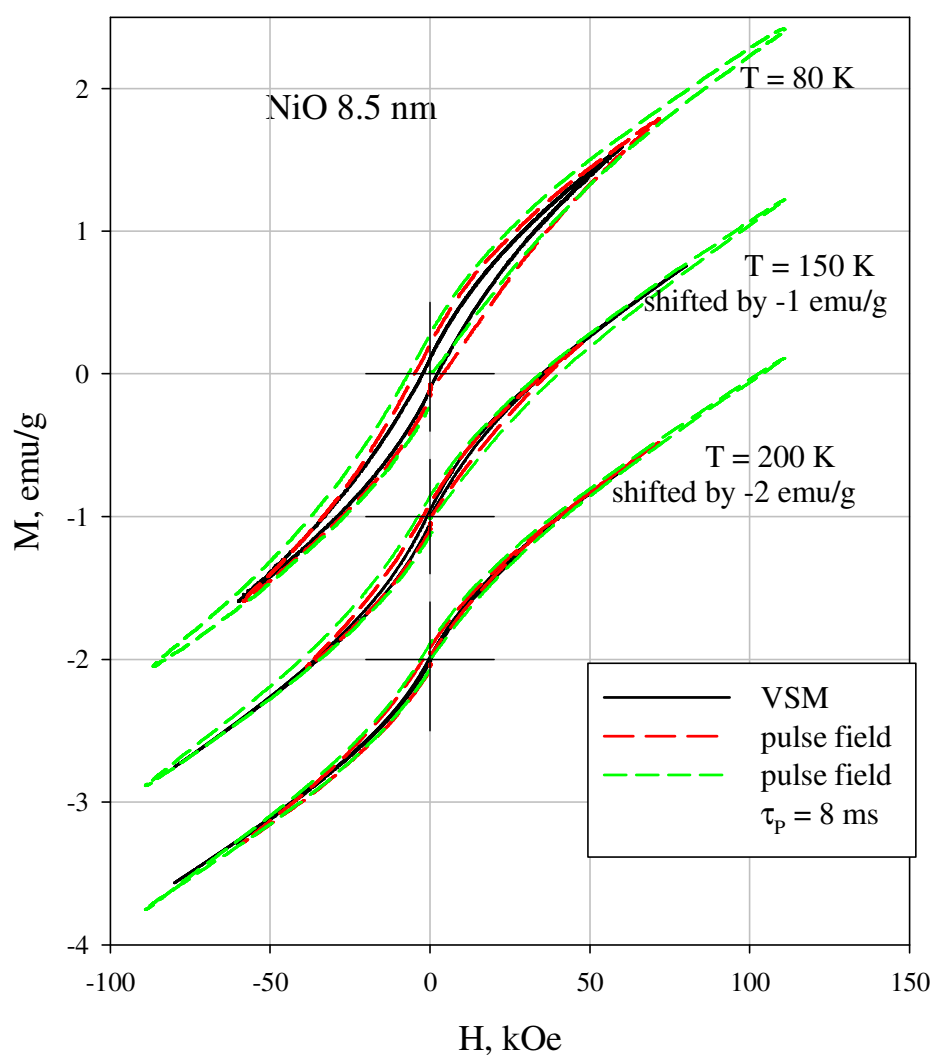

Fig. 7. Typical $M(H)$ dependences for $\mathrm{NiO}$ nanoparticles obtain in pulsed fields (dynamic hysteresis) and using the VSM technique (in fields of up to $60 \mathrm{kOe}$ ) at the indicated temperatures. The data obtained at 150 and $200 \mathrm{~K}$ are shifted along the ordinate axis by $-1 \mathrm{emu} / \mathrm{g}$ and $-2 \mathrm{emu} / \mathrm{g}$, respectively.

The measurements in pulsed fields (Fig. 3) detect portions of the hysteresis loops at fields (i) from $H=0$ to $H_{0}$, (ii) from $H_{0}$ to the negative field value somewhat lower than $\left|H_{0}\right|$ (damping oscillations in the LCR contour), and, then, (iii) down to the zero field. The most informative portion of the $M(H)$ dependence obtained using this technique is range (ii). According to harmonic law (1), which works with high accuracy in range (ii), the external field variation rate can be determined as a derivative of the $H(t)$ function. In the vicinity of $H=0$ and, at least, within $\pm 0.1 \mathrm{H}_{0}$, we have $\mathrm{dH} / \mathrm{dt} \approx$ const. Using Eq. (1), we obtain

$$
d H / d t=H_{0} / 2 \tau_{\mathrm{P}}
$$

The slopes of the straights in Fig. 3 correspond to the rate of the external field variation near $H=0$, which illustrates the aforesaid. The obtained coercivities were in the range of $\pm 0.1 \mathrm{H}_{0}$.

Figure 8 illustrates a typical behavior of the DMH loops obtained by the pulsed technique at different pulse parameters $\tau_{\mathrm{P}}$ and $H_{0}$ near the origin of coordinates (together with the VSM data). One can see the broadening of the hysteresis loops in range (ii) and a significant increase in the remanent magnetization both with increasing field $H_{0}$ and with decreasing pulse length $\tau_{\mathrm{P}}$. This behavior qualitatively reflects the expected effect of magnetization switching in an ac field. Below, we focus on the behavior of the coercivity $H_{\mathrm{C}}$ upon variation in the pulse parameters and temperature. 

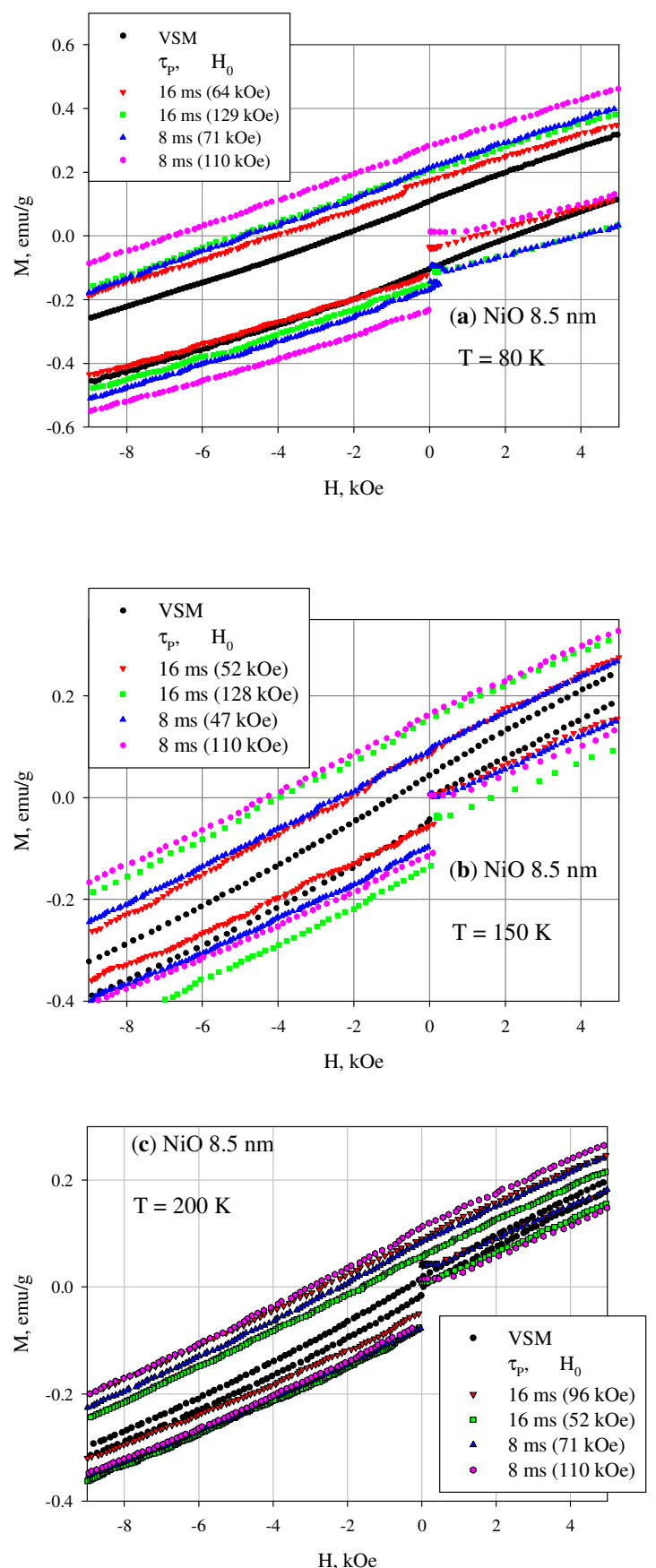

Fig. 8. Portions of the hysteresis loops obtained in pulsed fields (together with the VSM data) at different pulse parameters. The $\tau_{\mathrm{P}}$ and $\mathrm{H}_{0}$ values are shown in the figure.

\subsection{Dependence of the coercivity on the parameters of the pulsed field}

Both the $\tau_{\mathrm{P}}$ and $\mathrm{H}_{0}$ parameters, which were changed during the experiment, determine the magnetization switching rate $d H / d t$, in accordance with Eq. (2). The latter suggests that the growth of one of the parameters, e.g., doubling of the $H_{0}$ value, should have the same effect as halving of the other parameter $\left(\tau_{\mathrm{P}}\right)$. It is the picture that was observed upon pulsed field-induced magnetization switching in ferromagnetically ordered $\varepsilon-\mathrm{Fe}_{2} \mathrm{O}_{3}$ nanoparticles $[45,44]$ : the $H_{\mathrm{C}}(d H / d t)$ dependences for nanoparticles are single-valued $d H / d t$ functions. As was mentioned in Section 3.2, at temperatures of $80 \mathrm{~K}$ and higher, we can state that the magnetic hysteresis loops under the quasi-static conditions 
at $H_{0} \approx 70-80 \mathrm{kOe}$ are almost closed, while the $H_{\mathrm{C}}\left(H_{0}\right)$ dependences achieve a plateau (Fig. 6). Nevertheless, a unique $H_{\mathrm{C}}(d H / d t)$ dependence for $\mathrm{NiO}$ nanoparticles is not observed. This is illustrated in Figs. 9a and 9b, which show the $H_{\mathrm{C}}(d H / d t)$ and $H_{\mathrm{C}}\left(H_{0}\right)$ dependences, respectively. The $H_{\mathrm{C}}(d H / d t)$ dependence is divided into three separated clusters of points with the same pulse length $\tau_{\mathrm{P}}$. Moreover, when we plot the data in the coordinates $H_{\mathrm{C}}$ and $H_{0}$, the obtained $H_{\mathrm{C}}\left(H_{0}\right)$ dependences at $\tau_{\mathrm{P}}=$ const appear close to each other, although these are still three different $H_{\mathrm{C}}\left(H_{0}\right)$ dependences (Fig. $9 \mathrm{~b})$. According to our data, the described behavior is observed over almost the entire temperature range of 80-300 K where the dynamic hysteresis loops were measured.
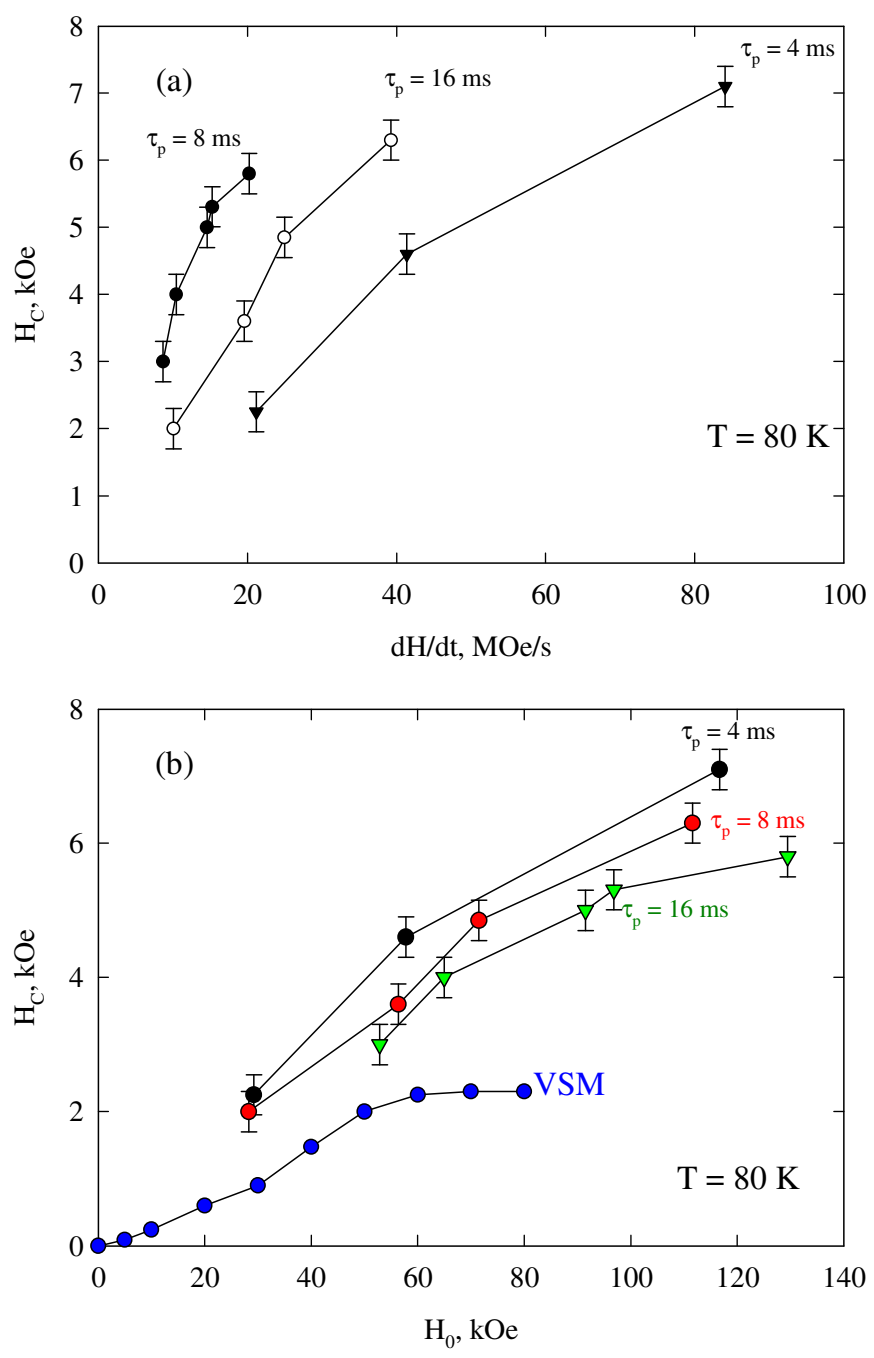

Fig. 9. Coercivity $H_{\mathrm{C}}$ vs (a) field variation rate $d H / d t$ and (b) maximum field $H_{0}$ upon pulsed magnetization switching in the $\mathrm{NiO}$ nanoparticle sample. Connecting lines group the data according to the pulse length $\tau_{\mathrm{P}}$. In (b), for comparison, the quasi-static magnetometry (VSM) data are presented.

Thus, the main difference between AFM nanoparticles and the previously investigated FM nanoparticles upon pulsed field-induced magnetization switching is the nonuniqueness of the functional $H_{\mathrm{C}}(d H / d t)$ dependence. In AFM nanoparticles, the maximum applied field $H_{0}$ plays the much more important role upon pulsed field-induced magnetization switching. Under these conditions, the magnetization curves clearer demonstrate their irreversibility (Fig. 7). It would be reasonable to attribute the observed behavior to the interaction of the magnetic subsystems in AFM 
nanoparticles, which manifests itself much brighter under the dynamic conditions, i.e., upon fast magnetization switching in strong fields. In principle, in the AFM particle, all the three possible magnetic subsystems, i.e., the AFM core, uncompensated moment (the FM subsystem), and surface spins, can interact. The AFM ordering exists over the entire investigated temperature range, since the Néel temperature, even with regard to its decrease for nanosized particles [67, 68], a priori goes beyond this range. If we take into account the paramagnetic state of surface spins at sufficiently high temperatures [10], then the «magnetic coupling» between the FM subsystem and the AFM core can make the main contribution to the observed behavior of the coercivity upon variation in the pulsed magnetization switching parameters.

The data presented in Fig. 9b show that the $H_{\mathrm{C}}\left(H_{0}\right)$ dependences under the pulsed fieldinduced magnetization switching have the negative curvature. Therefore, it is logical to assume that, similar to the case of the quasi-static conditions (Fig. 6), the $H_{\mathrm{C}}\left(H_{0}\right)$ dependences under the pulsedfield induced magnetization switching should also tend to a certain constant coercivity. This will be already a characteristic of the limiting hysteresis loop in the pulsed field, which obviously exceeds the $H_{\mathrm{C}}$ value for the limiting loop under the quasi-static conditions. As the temperature increases, the $H_{\mathrm{C}}\left(H_{0}\right)$ dependences become smoother (Fig. 10). This is also logical, since, with an increase in temperature, the «magnetic coupling» between the FM subsystem and the AFM core (or another magnetic subsystem) will weaken.

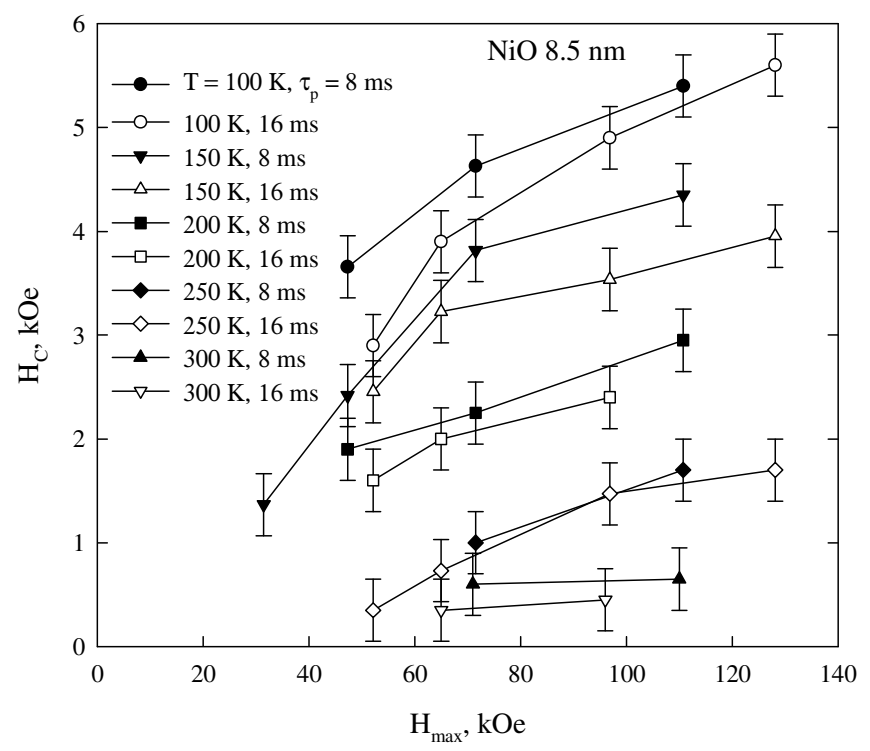

Fig. 10. Dependences of the coercivity $H_{\mathrm{C}}$ on the maximum applied field $H_{0}$ upon pulsed fieldinduced magnetization switching at the indicated pulse lengths $\tau_{\mathrm{P}}$ and temperatures.

\section{Temperature Evolution of the Coercivity for the Quasi-Static and Dynamic Hysteresis Loops}

Figure 11 shows temperature dependences of the coercivity for the quasi-static and pulsed field measurements. The data for the quasi-static magnetization switching conditions (VSM) were obtained in maximum fields of $80-90 \mathrm{kOe}$. Since the coercivity strongly depends on the pulse parameters, Fig. 10 presents the selected $H_{\mathrm{C}}(T)$ dependences measured in pulsed fields at the same parameters $H_{0}$ and $\tau_{\mathrm{P}}\left(H_{0}=65 \mathrm{kOe}, \tau_{\mathrm{P}}=16 \mathrm{~ms}, d H / d t=10 \mathrm{MOe} / \mathrm{s}\right.$ and $\mathrm{H}_{0}=110 \mathrm{kOe}, \tau_{\mathrm{P}}=8 \mathrm{~ms}$, $d H / d t=38 \mathrm{MOe} / \mathrm{s})$. It can be seen that the $H_{\mathrm{C}}(T)$ dependences for both the pulsed field-induced and quasi-static magnetization switching behave monotonically. This allows us to reliably extrapolate the experimental data to a value of $H_{\mathrm{C}}=0$. It was found that, at temperatures above $\sim 100 \mathrm{~K}$, the experimental $H_{\mathrm{C}}(T)$ dependences shown in Fig. 11 are satisfactorily described by the equation 


$$
H_{\mathrm{C}}(T)=H_{\mathrm{C}}{ }^{*} \cdot\left[1-\left(T / T_{\mathrm{B}}\right)^{0.5}\right] .
$$

Here, $H_{\mathrm{C}}{ }^{*}$ is the constant and $T_{\mathrm{B}}$ is the temperature at which the coercivity turns to zero. Obviously, the temperature $T_{\mathrm{B}}$ can be considered to be the superparamagnetic (SPM) blocking temperature; in this case, it should correspond to blocking (unblocking) of the particles with the greatest size. Lines in Fig. 10 are plotted using Eq. (3) at the $T_{\mathrm{B}}$ and $H_{\mathrm{C}}{ }^{*}$ values indicated in the figure caption.

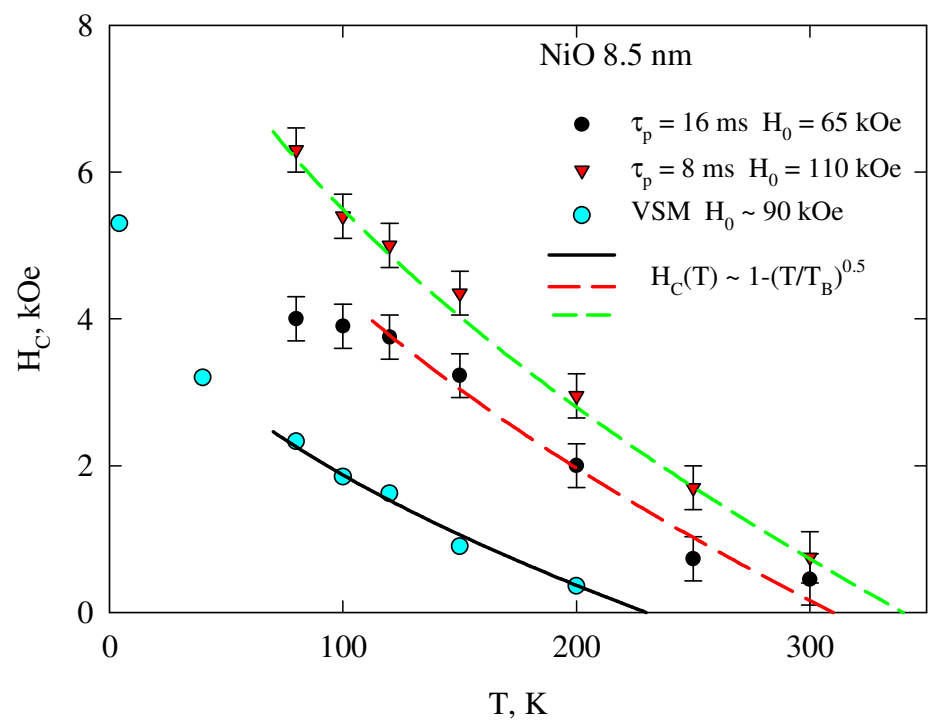

Fig. 11. Temperature dependences of the coercivity $H_{\mathrm{C}}$ obtained under the quasi-static conditions (VSM) and in pulsed fields at the indicated parameters of the pulse. Symbols correspond to the experiment. Solid lines are plotted using Eq. (3) at ${H_{\mathrm{C}}}{ }^{*}=5.5 \mathrm{kOe}$ and $T_{\mathrm{B}}=230 \mathrm{~K}$ for the VSM data, $H_{\mathrm{C}}{ }^{*}=10.5 \mathrm{kOe}$ and $T_{\mathrm{B}}=320 \mathrm{~K}$ for $\tau_{\mathrm{P}}=16 \mathrm{~ms}$, and $\mathrm{H}_{\mathrm{C}}{ }^{*}=12 \mathrm{kOe}$ and $T_{\mathrm{B}}=350 \mathrm{~K}$ for $\tau_{\mathrm{P}}=8 \mathrm{~ms}$.

Thus, for the case of the pulsed fields, there is a significant broadening of the region with $H_{\mathrm{C}} \neq 0$ in the $H_{\mathrm{C}}-T$ diagram. Let us analyze the obtained $T_{\mathrm{B}}$ values using the well-known equation for the SPM blocking:

$$
T_{\mathrm{B}}=K_{\mathrm{eff}} V / k_{\mathrm{B}} \ln \left(\tau_{\mathrm{m}} / \tau_{0}\right) .
$$

Here, $K_{\text {eff }}$ is the effective magnetic anisotropy constant, $V$ is the particle volume, $k_{\mathrm{B}}$ is the Boltzmann constant, and $\tau_{\mathrm{m}}$ and $\tau_{0}$ are the characteristic times of the measurement and relaxation of the particle magnetic moment, respectively. The relaxation time value $\tau_{0}$ can usually be within $10^{-9}-10^{-13} \mathrm{~s}$; for the quasi-static magnetic measurements, it is considered that $\tau_{\mathrm{m}} \sim 10^{2} \mathrm{~s}$ [2]. Obviously, the growth of the $T_{\mathrm{B}}$ value in the pulsed technique is caused by a decrease in the characteristic measuring time. Let us take $\tau_{0}=10^{-11} \mathrm{~s}$ and, for a maximum particle size of $d=15 \mathrm{~nm}$ from the distribution in Fig. 2 , we obtain using Eq. (4) that a value of $T_{\mathrm{B}}=230 \mathrm{~K}$ corresponds to the value of $\mathrm{K}_{\mathrm{eff}}=2.8 \times 10^{5} \mathrm{erg} / \mathrm{cm}^{3}$. At the same $\tau_{0}$ and $K_{\text {eff }}$ values, we can obtain from Eq. (4) that values of $T_{\mathrm{B}}=350 \mathrm{~K}$ and $320 \mathrm{~K}$ correspond to characteristic measuring times $\tau_{\mathrm{m}}$ of 3 and $21 \mathrm{~ms}$. These values are similar to pulse lengths $\tau_{\mathrm{P}}$ of 8 and $16 \mathrm{~ms}$, but not exactly equal to them. Here, it should be noted that, according to Eq. (4), the characteristic measuring time is not a time of measuring the hysteresis loop, which usually amounts to $\sim 10^{3} \mathrm{~s}$ in the quasi-static measurements [69]. Moreover, since the $H_{\mathrm{C}}$ value depends on the $H_{0}$ value (Figs. 8 and 9) in the pulsed measurements, it is difficult to point out a parameter uniquely related to $\tau_{\mathrm{m}}$. It is obvious, however, that the $\tau_{\mathrm{m}}$ and $\tau_{\mathrm{P}}$ values should be of the same order of magnitude. This is observed for the data obtained. 
It is worth noting that Eq. (3) with exponent $1 / 2$ or close to it is often used to describe the temperature dependence of the coercivity of single-domain FM nanoparticles [70-75] and, in this equation, we have $H_{\mathrm{C}}{ }^{*}=H_{\mathrm{C}}(T=0)$. It seems surprising that the data for the quasi-static measurements of AFM nanoparticles at temperatures from $80 \mathrm{~K}$ to $T_{\mathrm{B}}$ are satisfactorily described by the power law. The data for this temperature range can be considered corresponding to the limiting hysteresis loop (the $H_{\mathrm{C}}\left(H_{0}\right)$ dependences achieve a plateau, see Fig. 6). Nevertheless, at low (40 and $4.2 \mathrm{~K}$ ) temperatures, the $H_{\mathrm{C}}$ values obtained at the strong maximum field $H_{0}$, which corresponds already to the closed hysteresis loop, will obviously significantly increase. Therefore, functional dependence (3) does not describe the entire temperature range. The change in the functional temperature dependence of the coercivity can follow from switching of an additional source of the magnetic anisotropy. Such a mechanism can be the interaction with the subsystem of surface spins at the transition of this subsystem to the spin glass-like state [26-32] and, as rule, the temperature of freezing of the surface spin subsystem is lower than the SPM blocking temperature [26-32].

In addition, we have some comments concerning the $\mathrm{K}_{\text {eff }}$ value obtained above. A value of $T_{\mathrm{B}}=230 \mathrm{~K}$ determined from the quasi-static measurements at $H=0$ is similar to the temperature of the irreversible $M(T)$ behavior $\mathrm{T}_{\text {irr }} \approx 250 \mathrm{~K}$ (Fig. 4), which additionally confirms the reliability of the estimates made. The temperature $T_{\mathrm{irr}}$, as the temperature $T_{\mathrm{B}}$, under the condition $H=0$, corresponds to blocking of particles with the greatest size. A value of $K_{\text {eff }}=2.8 \mathrm{erg} / \mathrm{cm}^{3}$ noticeably exceeds the magnetic anisotropy constant $K_{\text {bulk }}=0.8 \times 10^{5} \mathrm{erg} / \mathrm{cm}^{3}$ of bulk nickel oxide [32]. This behavior observed frequently in FM nanoparticles is usually attributed to the manifestation of the surface magnetic anisotropy contribution [76-84, 45]. This contribution determined by the particle size is usually written in the form [79]

$$
K_{\text {eff }}=K_{\text {bulk }}+6 K_{\mathrm{S}} / d,
$$

where $K_{\mathrm{S}}$ is the surface magnetic anisotropy constant. Substituting the greatest particle size $d=15 \mathrm{~nm}$, which makes the maximum contribution to the coercivity, we can obtain the $\mathrm{K}_{\mathrm{S}}$ estimate, which amounts to $\sim 0.05 \mathrm{erg} / \mathrm{cm}^{2}$. A significant contribution of the surface magnetic anisotropy to the magnetic properties of AFM nanoparticles, including $\mathrm{NiO}$ ones, was mentioned in [85-88].

\section{Concluding remarks}

The magnetic properties of $\mathrm{NiO}$ nanoparticles investigated in this work reflect the existence and interaction of several magnetic subsystems in them. The linear growth of the magnetization in strong fields and the $M(H)$ slope larger than for bulk $\mathrm{NiO}$ evidence for the contribution of the subsystem of surface spins, along with the antiferromagnetically ordered particle core. The FM subsystem (the uncompensated magnetic moment) causes the behavior of the temperature dependences of the magnetization typical of the SPM blocking at different magnetic prehistories and the irreversible behavior of the $M(H)$ curves. However, the field of the irreversible behavior of the $M(H)$ dependence is fairly strong $\left(\sim 10^{5} \mathrm{Oe}\right)$ at low temperatures, although it decreases with increasing temperature. Only at temperatures about $80 \mathrm{~K}$ and higher, the conventionally used maximum applied field of $H_{0} \sim 80-90 \mathrm{kOe}$ becomes sufficient to obtain the magnetic hysteresis loop similar to the limiting (closed) loop. Consequently, the coercivity observed at low temperatures usually characterizes only a minor hysteresis loop. The $H_{\mathrm{C}}\left(H_{0}\right)$ dependences are $S$-shaped and, with increasing temperature (at $H_{0} \sim 80-90 \mathrm{kOe}$ ), the $H_{\mathrm{C}}$ value becomes similar to that for the limiting hysteresis loop. Under the magnetization switching induced by pulsed fields (up to maximum fields of $130 \mathrm{kOe}$ at pulse lengths of 4, 8, and $16 \mathrm{~ms}$ ), the described features of the magnetic hysteresis manifest themselves even brighter. The coercivity increases with a decrease in the pulse length and an increase in the $H_{0}$ value. In this case, the irreversibility of the magnetization curves is enhanced as compared with the quasi-static conditions. The main, striking fact observed in AFM nanoparticles is the nonuniqueness of the dependence of $H_{\mathrm{C}}$ on the external field variation rate $d H / d t$. According to the data obtained, the $H_{0}$ value affects the $H_{\mathrm{C}}$ value much stronger than in the case of FM nanoparticles. 
A set of the above-described properties typical of both the quasi-static and pulsed fieldinduced magnetization switching in $\mathrm{NiO}$ nanoparticles allows us to state that, in the case of AFM nanoparticles, there is a factor that significantly complicates the dynamics of magnetization switching in these objects as compared with the case of single-domain FM particles. This factor is the interaction between the magnetic subsystems in AFM nanoparticles. Under the dynamic magnetization switching, this interaction manifests itself stronger than under the quasi-static conditions. The interaction of the AFM core with the FM subsystem seems more logical, since the described behavior is characteristic of the temperature range up to room temperatures. However, based on the analysis of the temperature evolution of the quasi-static magnetic hysteresis loops, we can state the change in the functional $H_{\mathrm{C}}(T)$ dependence at temperatures below $80 \mathrm{~K}$. This can already be related to the interaction of the FM subsystem with surface spins at the transition of this subsystem to the spin glass state.

A significant broadening of the $H_{\mathrm{C}}-T$ diagram region where the coercivity is nonzero in the case of the pulsed fields used is satisfactorily explained by the SPM blocking, at which the temperature of the transition to the unblocked (blocked) state depends on the characteristic measuring time (the latter decreases by several orders of magnitude under the pulsed magnetization switching). This allowed us to estimate the additional contribution to the magnetic anisotropy. Under the assumption that this contribution is made by the effect of the size-dependent surface anisotropy, then the corresponding surface magnetic anisotropy constant $K_{\mathrm{S}}$ is $\sim 0.05 \mathrm{erg} / \mathrm{cm}^{2}$. This value is of the same order of magnitude as the value for the ferrimagnetic oxide nanoparticles.

Thus, the extremely complex character of magnetization switching in $\mathrm{NiO}$ nanoparticles under both the quasi-static and dynamic conditions reflects the interaction between the magnetic subsystems formed in such objects. This should be taken into account in the theoretical investigations of the DMH processes [51-53], since the data obtained in this work are characteristic of the entire class of AFM nanoparticles.

\section{Acknowledgments}

We are grateful to A.D. Balaev for fruitful discussions. The TEM study and magnetic measurements using a PPMS-9 facility were carried out on the equipment of the Krasnoyarsk Territorial Center for Collective Use, Krasnoyarsk Scientific Center, Siberian Branch, Russian Academy of Sciences.

1. L. Néel, C.R. Acad. Sci. Paris 252, 4075 (1961).

2. S. Mørup, D.E. Madsen, C. Fradsen, C.R.H. Bahl, and M.F. Hansen, J. Phys.: Condens. Matter 19, 213202 (2007).

3. Yu.L. Raikher and V.I. Stepanov, J. Phys.: Condens. Matter. 20, 204120 (2008).

4. E. Winkler, R.D. Zysler, M. Vasquez Mansilla, D. Fiorani, Phys. Rev. B 72, 132409 (2005).

5. S.D. Tiwari, K.P. Rajeev, Phys. Rev. B 72, 104433 (2005).

6. A.A. Lepeshev, I.V. Karpov, A.V. Ushakov, D.A. Balaev, A.A. Krasikov, A.A. Dubrovskiy, D.A. Velikanov, M.I. Petrov, J. Supercond. Nov. Magn. 30, 931 (2017).

7. S.A. Makhlouf, F.T. Parker, A.E. Berkowitz, Phys. Rev. B 55, R14717 (1997).

8. S.A. Makhlouf, F.T. Parker, F.E. Spada, and A.E. Berkowitz, J. Appl. Phys. 81 (8), 5561 (1997).

9. S.I. Popkov, A.A. Krasikov, A.A. Dubrovskiy, M.N. Volochaev, V.L. Kirillov, O.N. Martyanov, D.A. Balaev, J. Appl. Phys. 126, 103904 (2019). 
10. S.I. Popkov, A.A. Krasikov, D.A. Velikanov, V.L. Kirillov, O.N. Martyanov, D.A. Balaev, J. Magn. Magn. Mater. 483, 21 (2019).

11. M. Arif, A. Sanger, M. Shkir, A. Singh, R.S. Katiyar, Physica B 552, 88 (2019).

12. D. Nikolíc, M. Panjan, G.R. Blake, M. Tadić, Journal of the European Ceramic Society 35, 3843 (2015).

13. T. Tajiri,S. Saisho, M. Mito, H. Deguchi, K. Konishi, A. Kohno, J. Phys. Chem. C, 119, 11941200 (2015).

14. M. Tadić, M. Panjan, D. Marković, Materials Letters 64, 2129-2131 (2010).

15. J.F.K. Cooper, A. Ionescu, R.M. Langford, K.R.A. Ziebeck, C.H.W. Barnes, R. Gruar, C. Tighe, J.A. Darr, N.T.K. Thanh, B. Ouladdiaf, J. Appl. Phys. 114, 083906 (2013).

16. N.J.O. Silva, V.S. Amaral, and L.D. Carlos, Phys. Rev. B 71, 184408 (2005).

17. S.D. Tiwari, K.P. Rajeev, Solid State Communications 152, 1080 (2012).

18. D.A. Balaev, A.A. Dubrovskii, A.A. Krasikov, S.V. Stolyar, R.S. Iskhakov, V.P. Ladygina, and E.D. Khilazheva, JETP Lett. 98 (3), 139 (2013).

19. Ch. Rani, S.D. Tiwari, Appl. Phys. A 123, 532 (2017).

20. D.A. Balaev, A.A. Krasikov, A.A. Dubrovskiy, S.I. Popkov, S.V. Stolyar, R.S. Iskhakov, V.P. Ladygina, and R.N. Yaroslavtsev, J. Appl. Phys. 120, 183903 (2016).

21. D.A. Balaev, A.A. Dubrovskiy, A.A. Krasikov, S.I. Popkov, A.D. Balaev, K.A. Shaikhutdinov, V.L. Kirillov, and O. N. Mart'yanov, Physics of the Solid State, 59, (N8), 1547 (2017).

22. D.A. Balaev, A.A. Krasikov, S.V. Stolyar, R.S. Iskhakov, V.P. Ladygina, R.N. Yaroslavtsev, O.A. Bayukov, A.M. Vorotynov, M.N. Volochaev, and A.A. Dubrovskiy, Phys. Solid State 58 (2), 1782 (2016).

23. T. Iimori, Y. Imamoto, N. Uchida, Y. Kikuchi, K. Honda, T. Iwahashi, Y. Ouch, J. Appl. Phys. 127, 023902 (2020).

24. R.H. Kodama, A.E. Berkowitz, E.J. McNiff, and S. Foner, J. Appl. Phys. 81, 5552 (1997).

25. R.H. Kodama, A.E. Berkowitz, Phys. Rev. B 59, 6321 (1999).

26. E. Winkler, R.D. Zysler, M. Vasquez Mansilla, D Fiorani, D Rinaldi, M Vasilakaki, K.N. Trohidou, Nanotechnology 19, 185702 (2008).

27. A. Punnoose, T. Phanthavady, M.S. Seehra, N. Shah, and G.P. Huffman, Phys. Rev. B 69, 054425 (2004).

28. M.S. Seehra, V. Singh, X. Song, S. Bali, E.M. Eyring, J. Phys. Chem. Solids 711362 (2010).

29. M. Tadić, M. Panjan, D. Marković, I. Milošević, V. Spasojević, Journal of Alloys and Compounds 509, 7134 (2011).

30. N. Rinaldi-Montes, P. Gorria, D. Martínez-Blanco, A.B. Fuertes, L. Fernández Barquín, I. Puente-Orench, J.A. Blanco, Nanotechnology 26, 305705 (2015).

31. M. Tadic, D. Nikolic, M. Panjan, and G. R. Blake, Journal of Alloys and Compounds 647, 1061 (2015).

32. S.V. Stolyar, D.A. Balaev, V.P. Ladygina, A.I. Pankrats, R.N. Yaroslavtsev, D.A. Velikanov, R.S. Iskhakov, JETP Letters 111, 183 (2020).

33. I. Hrianca, C. Caizer, Z. Schlett, J. Appl. Phys. 92, 2125 (2002).

34. C. Caizer, I. Hrianca, Eur. Phys. J. B 31, 391 (2003). 
35. I. S. Poperechny, Yu. L. Raikher, and V. I. Stepanov, Phys. Rev. B 82, 174423 (2010).

36. S. Poperechny and Yu. L. Raikher, Physica B 435, 58 (2014).

37. Y.P. Kalmykov, S.V. Titov, W.T. Coffey, M. Zarifakis, W.J. Dowling, Phys. Rev. B 99, 184414 (2019).

38. E.L. Verde, G.T. Landi, J.A. Gomes, M.H. Sousa, A.F. Bakuzis, J. Appl. Phys. 111, 123902 (2012).

39. Y. Lv, Y. Yang, J. Fang, H. Zhang, E. Peng, X. Liu, W. Xiao, J. Ding, RSC Adv. 5, 76764 (2015).

40. E. Garaio, O. Sandre, J.-M. Collantes, J.A. Garcia, S. Mornet, F. Plazaola, Nanotechnology 26 (1), 015704 (2015).

41. A.S. Kamzin, A.A. Valiullin, H. Khurshid, Z. Nemati, H. Srikanth, and M.H. Phan, Physics of the Solid State 60, 382 (2018).

42. A.R. Sebastian, H.J. Kim, S.H. Kim, J. Appl. Phys. 126, 134902 (2019).

43. A.S. Kamzin, A.A. Valiullin, V.G. Semenov, H. Das, N. Wakiy, Physics of the Solid State 61, 1113 (2019).

44. S.I. Popkov, A.A. Krasikov, S.V. Semenov, A.A. Dubrovskii, S.S. Yakushkin, V.L. Kirillov, O.N. Mart'yanov, D.A. Balaev, Physics of the Solid State 62, 445 (2020).

45. D.A. Balaev, I.S. Poperechny, A.A. Krasikov, K.A. Shaikhutdinov, A.A. Dubrovskiy, S.I. Popkov, A.D. Balaev, S.S. Yakushkin, G.A. Bukhtiyarova, O.N. Martyanov, and Yu.L. Raikher, J. Appl. Phys. 117, 063908 (2015).

46. N.J.O. Silva, V.S. Amaral, A. Urtizberea, R. Bustamante, A. Millan, F. Palacio, E. Kampert and U. Zeitler, S. de Brion, O. Iglesias and A. Labarta, Phys. Rev. B 84, 104427 (2011).

47. N. A. Usov, Yu. B. Grebenshchikov, J. Appl. Phys. 106, 023917 (2009).

48. J. Carrey, B. Mehdaoui, M. Respaud, J. Appl. Phys. 109, 083921 (2011).

49. A.M. Shutyi, D.I. Sementsov, Phys. Solid State 61, 1736 (2019).

50. A.M. Shutyi, D.I. Sementsov, J. Exp. Theor. Phys. 129, 248 (2019).

51. B. Ouari, S. Aktaou, and Y.P. Kalmykov, Phys. Rev. B 81, 024412 (2010).

52. B. Ouari, Y.P. Kalmykov, Phys. Rev. B 83, 064406 (2011).

53. Yu.P. Kalmykov, B. Ouari, S.V. Titov, J. Appl. Phys. 120, 053901 (2016).

54. D.A. Balaev, A.A. Krasikov, A.A. Dubrovskiy, S.V. Semenov, S.I. Popkov, S.V. Stolyar, R.S. Iskhakov, V.P. Ladygina, and R.N. Yaroslavtsev, Phys. Solid State 58 (2) 287 (2016).

55. D.A. Balaev, A.A. Krasikov, A.A. Dubrovskii, A.D. Balaev, S.I. Popkov, V.L. Kirillov, O.N. Martyanov. J. Supercond. Nov. Magn. 32, 405 (2019).

56. D.A. Balaev, A.A. Krasikov, D.A. Velikanov, S.I. Popkov, N.V. Dubynin, S.V. Stolyar, V.P. Ladygina, R.N. Yaroslavtsev, Physics of the Solid State, 60, No. 10, 1973 (2018)

57. D.A. Velikanov, RF Patent No. 2481591C1, Byull. Izobret. No. 13 (2013). http://www.fips.ru/Archive/PAT/2013FULL/2013.05.10/DOC/RUNWC1/000/000/002/481/5 91/DOCUMENT.PDF

58. A.D. Balaev, Yu.V. Boyarshinov, M.M. Karpenko, and B.P. Khrustalev, Prib. Tekh. Eksp., 3, 167 (1985).

59. A.A. Bykov, S.I. Popkov, A.M. Parshin, A.A. Krasikov, Journal of Surface Investigation. Xray, Synchrotron and Neutron Techniques 9, 111 (2015). 
60. S. Baran, A. Hoser, B. Penc, A. Szytuła, Acta Polonica A 129, 35 (2016)

61. H. Shim, P. Dutta, M.S. Seehra, J. Bonevich, Solid State Communications 145, 192 (2008).

62. C. Gilles, P. Bonville, H. Rakoto, J.M. Broto, K.K.W. Wong, S. Mann. J. Magn. Magn. Mater. 241, 430 (2002).

63. N.J.O. Silva, A. Millan, and F. Palacio, E. Kampert and U. Zeitler, V.S. Amaral, Phys. Rev. B 79, 104405 (2009).

64. R. D. Desautels, E. Skoropata, Y.-Y. Chen, H. Ouyang, J. W. Freeland, and J. van Lierop, J. Phys.: Condens. Matter 24, 146001 (2012).

65. A. Cabot, P. Alivisatos, W. F. Puntes, L. Balcells, O. Iglesias, and A. Labarta, Phys. Rev. B 79, 094419 (2009).

66. E. Winkler, R. D. Zysler, and D. Fiorani, Phys. Rev. B 70, 174406 (2004).

67. S. Thota, J.H. Shim, M.S. Seehra, J. Appl. Phys. 114, 214307 (2013).

68. N. Rinaldi-Montes, P. Gorria, D. Martínez-Blanco, A.B. Fuertes, I. Puente-Orench, L. Olivi, J.A. Blanco, AIP Advances 6, 056104 (2016).

69. Assuming $\tau_{\mathrm{m}}$ to be the time of measuring $3 / 4$ of the hysteresis loop and taking $\tau_{\mathrm{m}}=1.5 \times 10^{3} \mathrm{~s}$, $24 \mathrm{~ms}$, and $12 \mathrm{~ms}$ for $T_{\mathrm{B}}=230 \mathrm{~K}, 320 \mathrm{~K}$, and $350 \mathrm{~K}$ respectively, we obtain that the $\mathrm{K}_{\text {eff }}$ value lies between $(2.9-3.05) \times 10^{5} \mathrm{erg} / \mathrm{cm}^{3}$, which is similar to $2.8 \times 10^{5} \mathrm{erg} / \mathrm{cm}^{3}$.

70. L. Néel. Ann. Geophysique 5, 99 (1949).

71. W.F. Brown. Phys. Rev. 130, 1677 (1963).

72. S.V. Komogortsev, R.S. Iskhakov, A.D. Balaev, A.V. Okotrub, A.G. Kudashov, N.A. Momot, and S.I. Smirnov, Phys. Solid State 51, 2286 (2009).

73. S.V. Komogortsev, R.S. Iskhakov, A.D. Balaev, A.G. Kudashov, A.V. Okotrub, S.I. Smirnov, Phys. Solid State 49, 734 (2007).

74. D.A. Balaev, S.V. Semenov, A.A. Dubrovskii, A.A. Krasikov, S.I. Popkov, S.S. Yakushkin, V.L. Killov, O.N. Mart'yanov, Physics of the Solid State 62, 285 (2020).

75. J.M. Vargas, A. Srivastava, A. Yourdkhani, L. Zaldivar, G. Caruntu, L. Spinu, J. Appl. Phys. 110, 064304 (2011).

76. A. Aharoni, J. Appl. Phys. 61, 3302 (1987).

77. V.P. Shilov, J.-C. Bacri, F. Gazeau, F. Gendron, R. Perzynski, Y.L. Raikher, J. Appl. Phys. 85, 6642 (1999).

78. X. Batlle, A. Labarta, J. Phys. D: Appl. Phys. 35, R15 (2002)

79. F. Bødker, S. Mørup, S. Linderoth, Phys. Rev. Lett. 72, 282 (1994).

80. C.-R. Lin, R.-K. Chiang, J.-S. Wang, T.-W. Sung, J. Appl. Phys. 99 08N710 (2006).

81. D.A. Balaev, S.V. Semenov, A.A. Dubrovskiy, S.S. Yakushkin, V.L. Kirillov, O.N. Martyanov, J. Magn. Magn. Mater. 440199 (2017).

82. Yu.V. Knyazev, D.A. Balaev, V.L. Kirillov, O.A. Bayukov, O.N. Mart'yanov, JETP Letters, 108, 527 (2018).

83. I.G. Vazhenina, R.S. Iskhakov, L.A. Chekanova, Phys. Solid State 60, 292 (2018).

84. P. Allia, G. Barrera, P. Tiberto, T. Nardi, Y. Leterrier, M. Sangermano, J. Appl. Phys. 116, 113903 (2014). 
85. D.A. Balaev, A.A. Krasikov, A.A. Dubrovskiy, S.I. Popkov, S.V. Stolyar, O.A. Bayukov, R.S. Iskhakov, V.P. Ladygina, R.N. Yaroslavtsev. J. Magn. Magn. Mater. 410, 71 (2016).

86. E.L. Duarte, R. Itri, E. Lima Jr, M.S. Baptista, T.S. Berquó, G.F. Goya, Nano- technology 17, 5549 (2006).

87. T.S. Berquó, J.J. Erbs, A. Lindquist, R.L. Penn, S.K. Banerjee, J. Phys.: Condens. Matter. 21, 176005 (2009).

88. M.P. Proenca, C.T. Sousa, A.M. Pereira, P. B. Tavares, J. Ventura, M. Vazquez, J.P. Araujo, Phys. Chem. Chem. Phys., 13, 9561 (2011). 